\title{
Dysphagia in Wilson's Disease: A Case Report of One-Year Follow-Up
}

\author{
Doyoung Kim, M.D., Yoon Ghil Park, M.D., Ph.D., Jung Hyun Park, M.D., Ph.D., \\ Jinyoung Park, M.D. \\ Department of Rehabilitation Medicine, Gangnam Severance Hospital, Rehabilitation Institute of Neuromuscular \\ Disease, Yonsei University College of Medicine, Seoul, Korea
}

\begin{abstract}
Wilson's disease (WD) is a genetic disease caused by an ATP7B gene mutation. Although dysphagia is known as a neurological manifestation of $\mathrm{WD}$, clinical case reports with post-treatment long-term follow-up are scarce. A 17year-old male was admitted to hospital complaining of general weakness and swallowing difficulty. He was diagnosed with WD by genetic confirmation. Assessment of the videofluoroscopic swallowing study (VFSS) determined an initial videofluoroscopic dysphagia scale (VDS) score of 48. After 11 months treatment with D-penicillamine and neuromuscular electrical stimulation therapy (NMES) with oromotor exercises, the VDS score improved to 23, especially in the following areas: mastication, apraxia, premature bolus loss, triggering of pharyngeal swallow, and laryngeal elevation. Dysphagia is an early neurological symptom of WD, and is reversible when properly treated with early diagnosis. Thus, since WD is treatable, patients presenting with dysphagia should be indicative of high probability of the disease, and evaluated at the earliest. NMES treatment in combination with D-penicillamine helps to improve the deglutition function in both oral and pharyngeal phases. To investigate the characteristics of dysphagia and the specific clinical efficacy of NMES in WD, further studies with larger number of patients are required. (JKDS 2021;11:82-86)
\end{abstract}

Keywords: Wilson's disease, Deglutition disorders, Electrical stimulation

\section{INTRODUCTION}

Wilson's disease (WD) is a genetic disease caused by an ATP7B gene mutation. Dysfunction of coppertransporting P-type ATPase causes accumulation of copper, which leads to multiple organ failure. Several neurologic symptoms may develop when copper accumulates in the cerebral tissue. In WD, patients with brain stem lesion has been reported to have dys- phagia as a main neurological symptom ${ }^{1}$. Along with medication, there have been several studies which revealed the usefulness of neuromuscular electrical stimulation (NMES) in dysphagia of $\mathrm{WD}^{1,2}$. However, these studies lack detailed analysis of which specific swallowing process malfunctions and improves with treatment. This present study reports on a WD patient with dysphagia, which showed improvement in specific swallowing process. This is a long-term follow
Received: July 20 2020, Revised: July 21 2020,

Accepted: September 32020

Corresponding author: Jinyoung Park, Department of Rehabilitation Medicine, Gangnam Severance Hospital, Yonsei University, 20 Eonju-ro 63-gil, Gangnam-gu, Seoul 06229, Korea Tel: +82-2-2019-3498, Fax: +82-2-2019-4881

E-mail: pjyblue511@gmail.com
Copyrights (c) The Korean Dysphagia Society, 2021. 
up study, which confirmed the improvement in swallowing function and neuro-imaging signs.

\section{CASE REPORT}

A 17-year-old male with no specific medical or family history was admitted to the department of pediatrics in a tertiary hospital for chief complaints of general weakness, tremor in the toes of both feet, speech disorder, and swallowing difficulty that has been worsening over the course of a month. KayserFleischer rings were seen in both eyes. The result of muscle strength testing using the Medical Research Council (MRC) scale was grade 2 in the upper extremities and grade 3 in the lower extremities bilaterally. The patient showed dysdiadochokinesia in both arms along with hand tremor. Motor aphasia with articulation disorder and dysphagia were also observed. Based on the clinical findings of an appreciably delayed oral phase in swallowing, a parenteral liquid diet was initiated via a nasogastric tube. Laboratory tests showed decreased serum concentrations of copper $(28.4 \mu \mathrm{g} / \mathrm{dL}$; normal range: 75.0-145.0 $\mu \mathrm{g} / \mathrm{dL}$ ) and ceruloplasmin ( $<4 \mathrm{mg} / \mathrm{dL}$; normal range: $16.2-35.6 \mathrm{mg} / \mathrm{dL})$. The 24-hour urine collection test showed an increased urine copper excretion (2993.5 $\mu \mathrm{g} /$ day; normal range: 15.0-60.0 $\mu \mathrm{g} /$ day). Genetic testing, which was conducted under the clinical impression of WD, confirmed exon 8 duplication, and substitution mutations of the ATP7B gene. He was diagnosed with WD by genetic confirmation, and brain magnetic resonance imaging (MRI) showed increased signal intensity in the pons and midbrain on diffusion-weighted imaging (DWI).(Fig. 1A, B) Video-
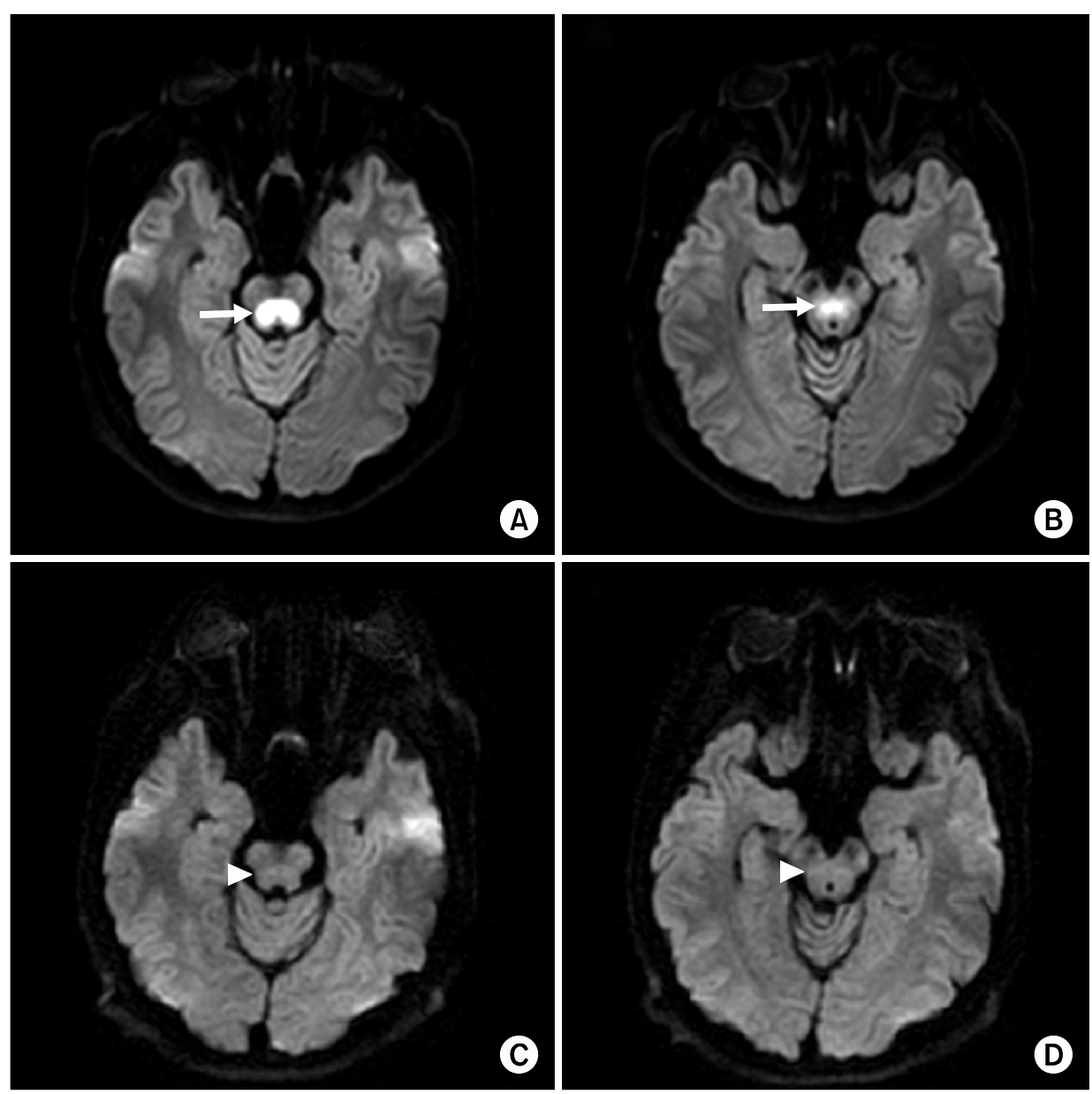

Fig. 1. One-year follow-up brain magnetic resonance imaging (MRI) on diffusion weighted image (DWI). The initial brain MRI shows high signal intensities (white arrow) in the pons and midbrain (A, B). The 1-year follow-up brain MRI showed decreased signal intensities (white arrow head) in the pons and midbrain (C, D). 
fluoroscopic swallowing study (VFSS) was performed to detect structural and functional problems in the oropharyngeal swallowing process using radiological fluoroscopy (SF-VA2000 [Prius-C Ergo]; HITACHI, Japan). As the patient was in an upright sitting position on a chair, the lateral view of the entire swallowing process was recorded and encoded as a video clip (MPG file with 30 frames/second). He was given the test foods, which were mixed with barium, in the following order: 1) $5 \mathrm{~mL}$ of solid, 2) $5 \mathrm{~mL}$ of semisolid, 3) $5 \mathrm{~mL}$ of semi-liquid, 4) $5 \mathrm{~mL}$ of liquid, and 5) $15 \mathrm{~mL}$ of liquid. The solid, semi-solid, semi-liquid, and liquid corresponded to level 7 (regular food), 4 (pureed food), level 2 (mildly thick drink) and level 0 (thin drink) according to the international dysphagia diet standardization initiative (IDDSI) framework. The dysfunctions in the oral phase included reduced tongue movement, decreased bolus formation, and prolonged oral transit time (OTT) in all formulas. In the pharyngeal phase, with a delayed swallowing reflex and decreased laryngeal elevation, the residue was detected in the vallecular space and pyriform sinus in all formulas. During 5 or $15 \mathrm{~mL}$ liquid swallowing, laryngeal penetration was detected, and the penetration aspiration scale (PAS) score was 3 . The test foods other than liquid did not progress into the pharynx due to the great delay in the oral phase. The videofluoroscopic dysphagia scale (VDS) score was 48.(Table 1) For treatment of dysphagia, the NMES was applied to the suprahyoid muscles bilaterally to assist laryngeal elevation using (VitalStim ${ }^{\circledR}$, Chattanooga Group, USA). The active electrodes were placed on the midpoint between the chin and the upper border of the hyoid bone. The reference electrodes were placed on the midpoint of the mandibular edge between the mental protuberance and bilateral mandibular angles. 15 sessions of NMES were carried out (30 minutes/session/day) with the following setting: frequency of $80 \mathrm{~Hz}$, amplitude of $6.5 \mathrm{~mA}$, and phase duration of $700 \mathrm{~ms}$. In addition, the patient was educated on several compensating methods, including 'chin tuck' and 'double swallowing', and trained with the oromotor exercises to facilitate the mobility and
Table 1. One-year follow-up of videofluoroscopic swallowing study.

\begin{tabular}{lcc}
\hline \multicolumn{1}{c}{ Parameters of VDS } & $\begin{array}{c}\text { Initial VFSS } \\
\text { before treatment } \\
\text { (February } 12, \\
\text { 2016) }\end{array}$ & $\begin{array}{c}\text { Follow-up VFSS } \\
\text { after } \\
\text { (January 13, } \\
\text { 2017) }\end{array}$ \\
\hline Lip closure & 2 & 2 \\
Bolus formation & 3 & 3 \\
Mastication* & 4 & 0 \\
Apraxia* & 3 & 0 \\
Tongue-to-palate contact & 5 & 5 \\
Premature bolus loss* & 4.5 & 3 \\
Oral transit time* & 3 & 0 \\
Triggering of pharyngeal swallow* & 4.5 & 0 \\
Vallecular residue & 4 & 4 \\
Laryngeal elevation* & 9 & 0 \\
Pyriform sinus residue & 0 & 0 \\
Coating on the pharyngeal wall & 0 & 0 \\
Pharyngeal transit time & 0 & 0 \\
Aspiration & 6 & 6 \\
Total VDS score & 48 & 23 \\
\hline
\end{tabular}

*Improved parameter in the follow-up VFSS.

VFSS: videofluoroscopic swallowing study, VDS: videofluoroscopic dysphagia scale.

the strength of the lips, tongue, and jaw, to be performed twice a day. D-penicillamine, pyridoxine, calcium pantothenate, nicotinamide, prednisolone, vitamin complex were prescribed and continued after discharge through regular outpatient follow-ups. After a short-term dysphagia rehabilitation during hospitalization, there was no significant improvement in dysphagia symptom. Especially, the aspiration symptoms were sustained, so that the follow-up VFSS was not tried during hospitalization. Therefore, at the time of discharge, the liquid diet was maintained through the nasogastric tube without oral try. After transferring to other rehabilitation hospital, the dysphagia rehabilitation program of the same protocol which was performed in our institute, including oromotor exercises and NMES was continued 5 times a week. After 11 months later, he was re-admitted to our institute for a regular check-up. Nevertheless of the decreased motor score in the lower extremities (from grade 3 to 2), the deglutition function had improved, so the diet level was built up to general diet and liquid with thickner. In the follow-up VFSS, the VDS score improved to 23. (Table 1) In the oral phase, the OTT had improved to less than $1 \mathrm{~s}$. In the pharyngeal 

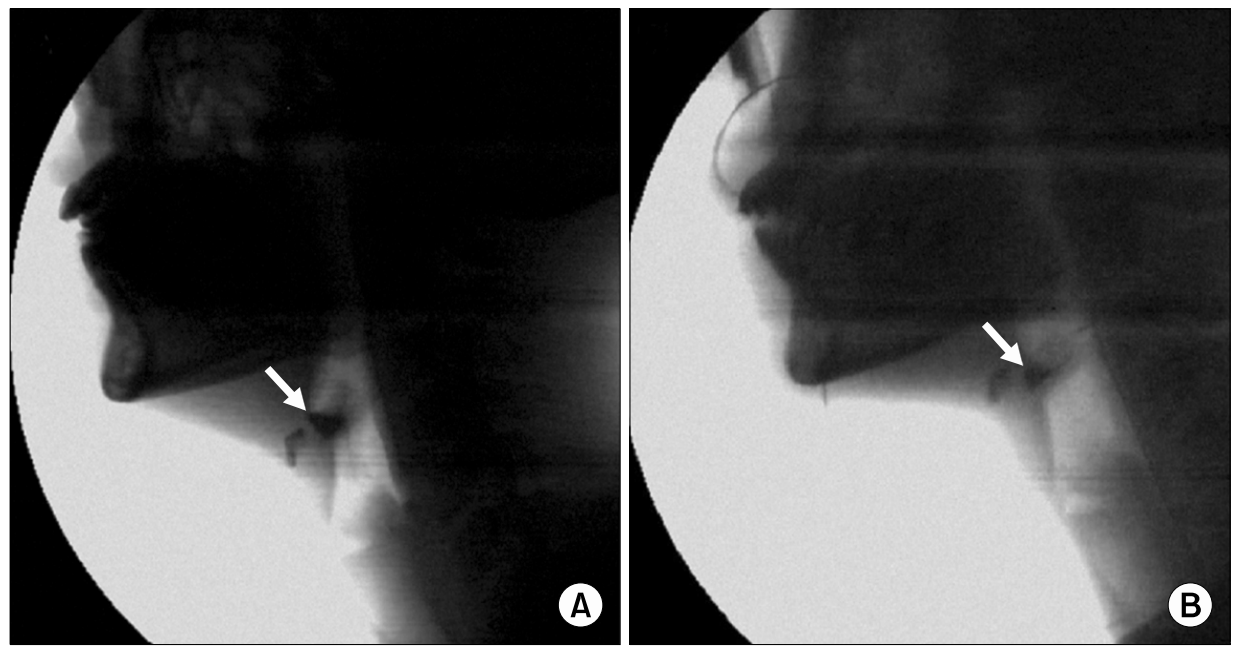

Fig. 2. One-year follow-up videofluoroscopic swallowing study (VFSS). The amount of semisolid vallecular residue (B) was reduced compared to the initial VFSS (A). The white arrow is the vallecular residue.

phase, the amount of residues in the vallecular space and pyriform sinus were decreased compared to that in the initial VFSS.(Fig. 2) Laryngeal penetration was observed only with $5 \mathrm{~mL}$ of liquid food, and the PAS score was improved to 2 . In the follow-up brain MRI, the increased signal intensities initially observed in the pons and midbrain on DWI had significantly resolved.(Fig. 1C, D) This case report was approved by the institutional review board (3-2019-0406).

\section{DISCUSSION}

The swallowing process is thought to be the result of a complex interaction between the cerebral cortex and brainstem motor or sensory nuclei. Thus, lesions that affect these structures may affect swallowing mechanisms and cause neurogenic dysphagia. In the present study, brainstem lesions of WD, including those that involve the pons and the midbrain, caused increased signal intensities on MRI due to copper accumulation. A significant increase in tissue copper content in the central nervous system causes damage to cellular structures, and it is thought that the site of copper deposition and the neurological symptoms may be correlated. The pons and midbrain constitute the ascending reticular activating system, which relays the signal produced in a central pattern generator (CPG), so that it functions to help rhythmic swallowing. In addition, the sensory feedback is believed to be conveyed to the cortical area via a first relay in the pons. In particular, pontine neurons, which play a role in transmitting sensory signals, are thought to be involved in the transfer of information from the oropharyngeal receptors to the higher centers in the central nervous system ${ }^{3}$. The ponto-corticomedullary loops might control the activity of the CPG swallowing neurons. If there is a problem with loop signal transmission, the swallowing process may get stuck at the oropharyngeal phase or at the early esophageal phase ${ }^{4}$. Based on these results, the improvement of dysphagia symptoms in this present study correlates the resolution of the anatomical lesion in neuroimaging, which may have resulted from the medication. It was not clear whether the improvement of dysphagia at the follow-up session after 1 year was due to the effect of D-penicillamine treatment or the rehabilitation program for dysphagia, including NMES because these treatments were administered concurrently. However, the improvement of dysphagia symptoms despite the progressed weakness in lower limbs suggests that the rehabilitation program for dysphagia, including NMES, was specifically effective in treating dysphagia. This focal symptom improvement is more likely due to the specific dysphagia rehabilitation rather than a medication effect, which is a systemic treatment. This case suggests that dysphagia in WD can be selectively reversible with early detection and treatment. There have been no studies 
which showed improvement after NMES borne out by analyzing each detailed step of the swallowing process. The efficacy of NMES observed in this case is thought to be mediated by two mechanisms. First, sensory stimulation may increase local sensory input to the central nervous system via the CPG, which is located in the lower brainstem. It may also have a long-term effect in reorganization of the cerebral cortex, resulting in the enhancement of brain plasticity and recovery in control of swallowing ${ }^{5-7}$. Second, motor stimulation may provoke contraction of pharyngeal muscles such as the suprahyoid muscle, which may improve laryngeal elevation and avoid disuse atrophy of muscle ${ }^{8-10}$. Sensory stimulation and muscle contraction aided by NMES helps reconstruct the control function of the brainstem reflex center over the swallowing reflex and enhances pharyngeal muscle coordination ${ }^{2}$. In this present case, the oromotor exercise seems to have been effective in promoting tongue movement and mastication, shortening the OTT and allowing the bolus transition from the oral cavity to the pharynx. NMES may have helped improve the triggering of pharyngeal swallow and laryngeal elevation. From now on, no study has confirmed improvement of the symptoms of dysphagia in WD after a long treatment period. This case report is meaningful in that it evaluated the scale of dysphagia in detail and checked the symptoms of the patient for a prolonged time of 1 year. A high index of suspicion for dysphagia in WD is necessary since it is reversible when properly treated under early diagnosis. An active rehabilitation program for dysphagia, including oromotor exercise and NMES, should be considered as a strong option for the treatment of dysphagia in patients with WD. Further studies with more cases are necessary to investigate the characteristics of dysphagia and the clinical efficacy of NMES in WD.

\section{CONFLICT OF INTEREST}

The authors declare that they have no competing interests. None of the authors have any conflicts of interest to disclose.

\section{ACKNOWLEDGEMENTS}

None of the authors had any funding to support this case report.

\section{REFERENCES}

1. Lee SY, Yang HE, Yang HS, Lee SH, Jeung HW, Park YO. Neuromuscular Electrical Stimulation Therapy for Dysphagia Caused by Wilson's Disease. Ann Rehabil Med. 2012;36:409-413.

2. Li X, Li L. Efficacy of neuromuscular electrical stimulation in Wilson's disease patients with dysphagia. J Phys Ther Sci. 2019;31:971-4.

3. Jean A. Brain stem control of swallowing: neuronal network and cellular mechanisms. Physiol Rev. 2001;81:92969.

4. Jean A, Car A. Inputs to the swallowing medullary neurons from the peripheral afferent fibers and the swallowing cortical area. Brain Res. 1979;178:567-72.

5. Hamdy S, Rothwell JC, Aziz Q, Singh KD, Thompson DG. Long-term reorganization of human motor cortex driven by short-term sensory stimulation. Nat Neurosci. 1998;1: 64-8.

6. Hamdy S, Rothwell JC, Aziz Q, Thompson DG. Organization and reorganization of human swallowing motor cortex: implications for recovery after stroke. Clin Sci (Lond). 2000;99:151-7.

7. Steele CM, Miller AJ. Sensory input pathways and mechanisms in swallowing: a review. Dysphagia. 2010;25:32333.

8. Freed ML, Freed L, Chatburn RL, Christian M. Electrical stimulation for swallowing disorders caused by stroke. Respir Care. 2001;46:466-74.

9. Lim KB, Lee HJ, Yoo J, Kwon YG. Effect of Low-Frequency rTMS and NMES on Subacute Unilateral Hemispheric Stroke With Dysphagia. Ann Rehabil Med. 2014;38:592602.

10. Blumenfeld L, Hahn Y, Lepage A, Leonard R, Belafsky PC. Transcutaneous electrical stimulation versus traditional dysphagia therapy: a nonconcurrent cohort study. Otolaryngol Head Neck Surg. 2006;135:754-7. 